\title{
No significant differences between conservative interventions and surgical interventions for TMJ disc displacement without reduction
}

\author{
Abstracted from \\ Al-Baghdadi M, Durham J, Araujo-Soares V, Robalino S, Errington L, Steele J. \\ TMJ Disc Displacement without Reduction Management: A Systematic Review. \\ J Dent Res 2014; 93: 37S-51S. [Epub ahead of print] PubMed PMID: 24659775. \\ Address for correspondence: Dr. M Al-Baghdadi, Department of Oral and Maxillofacial Surgery, \\ School of Dental Sciences, Newcastle University, UK. E-mail: m.k.s.al-baghdadi@ncl.ac.uk
}

\section{Question: In patients with temporomandibular joint (TMJ) disc displacement without reduction (DDwoR) are conservative or surgical interventions more effective?}

Data sources The Cochrane Central Register of Controlled Trials (CENTRAL), Medline, Embase and Scopus databases were searched. In addition reference lists of relevant review articles, textbook chapters and seven relevant journals were hand searched.

Study selection Randomised or quasi-randomised controlled trials in patients with clinical and/or radiological diagnosis of acute or chronic DDwoR undergoing any form of conservative or surgical intervention were considered. The primary outcomes were TMJ pain intensity and unassisted/active maximum mouth opening (MMO).

Data extraction and synthesis Study selection, data abstraction and quality assessment were conducted independently by two authors. The Cochrane risk of bias tool was used for the quality assessment. Data analysis was based on Cochrane statistical guidelines. For dichotomous data, the estimates of effect of an intervention were expressed as risk ratios (RR) together with $95 \%$ confidence intervals (Cl). For continuous data, mean differences (MD) with $95 \% \mathrm{Cl}$ were used.

Results Twenty studies involving a total of 1305 patients were included. Twelve studies were considered to be at high risk of bias with eight being at unclear risk of bias. There was a high degree of clinical heterogeneity among the studies included. Twenty-one comparisons were made among interventions. Meta-analyses were carried out for four comparisons. In most comparisons made there were no statistically significant differences between interventions relative to primary outcomes at short- or long-term follow-up.

Conclusions Most interventions appear to alleviate DDwoR symptoms, with no significant differences between non-invasive conservative interventions and minimally invasive or invasive surgical interventions. Given the paucity of evidence and the difficulty in interpreting the minimal clinically important difference, this finding suggests that patients with DDwoR probably should be initially managed with the most minimal and least invasive intervention. Escalation to more invasive treatment should occur only in the face of objective clinical need. This, however, should be interpreted in the context of a review based mostly on single studies of unclear to high risk of bias. Future well-conducted research may change or confirm this.

\section{Commentary}

The review assessed systematically all the published literature on the management of temporomandibular joint (TMJ) disc displacement without reduction (DDWoR). In the acute phase, such a condition is considered one of the main reasons for sudden-onset painful limitation of mouth opening. ${ }^{1}$ Over the years, suggested approaches to its management went through a paradigm shift from irreversible, invasive, mechanical therapies based on purported disc repositioning to more conservative strategies. ${ }^{2}$ By browsing four medical databases to search for any randomised clinical trials (RCTs) on the topic, the authors concluded that there is no evidence that more complex interventions have superior effectiveness over less invasive ones, thus suggesting that patients with DDWoR should be initially managed by the simplest approaches.

The systematic review was conducted according to recognised reference standards for data management (ie Cochrane Collaboration; Centre for Reviews and Dissemination) and reporting (ie PRISMA). Studies were included on the basis of welldescribed PICOS-like criteria. From a total of over 3000 citations, the reviewers selected 20 papers featuring a RCT or quasi-RCT design and investigating 21 comparisons between various treatment modalities. The treatments under assessment were very variable, ranging from conservative strategies (ie education, selfmanagement, oral appliances, physiotherapy, alone or combined) to minimally invasive interventions (ie arthrocentesis), to more invasive surgical procedures (ie arthroscopy, open surgery). Given the heterogeneity of the reviewed comparisons between those approaches, meta-analysis of findings was made only on four comparisons. The possibility to compare data between different studies was also limited by the very highly variable data at the patient- and disease-based level, due to the wide spectrum of age and sex distribution of the study population as well as the actual time of DDWoR occurrence.

In general, on the one hand, findings are supportive of good improvements with respect to baseline pain levels and mouth opening values for the majority of the reviewed interventions; on the other hand, they also showed equality of effectiveness between more and less invasive interventions. From a methodological viewpoint, the review is well-conducted, but its quality and the strength of conclusions are limited by the poor quality of the single reviewed papers. In particular, discussion of data in terms of their actual clinical implications could not be performed.

The review's suggestions that in patients with DDWoR conservative approaches should be attempted first are in line 


\section{TEMPOROMANDIBULAR JOINT DISORDERS}

with clinical empirical evidence that invasive interventions have a very limited place in the practice of TMJ disorders, as suggested by the favourable natural course of disease in TMD patients with low psychosocial impairment, ${ }^{3}$ as well as by the natural course of untreated DDWoR at 12 weeks. ${ }^{4}$ Thus, researchers attempting to get deeper into the issue of DDWoR management should bear in mind that factors such as the chronicity of the onset of mouth opening restriction and the patients' psychosocial profile likely play a pivotal role in explaining the evolution of symptoms, which are clearly not dependent on the disc position itself.

The main clinical implications that can be drawn from the reviewed literature is that, based on the unspecific effectiveness of various interventions, answers to the physiopathology of TMJ DDWoR should be searched within the behavior of jaw muscles, the antalgic co-contracture of which is likely to be mainly responsible for locking the mandible, instead of the commonly-believed biomechanical obstacle caused by the displaced disc.

Daniele Manfredini

Temporomandibular Disorders Clinic, Department of Maxillofacial Surgery, University of Padova, Italy

\section{Practice points}

- TMJ disc displacement without reduction seems to respond well to several treatment modalities, possibly suggesting the treatment effect is, at least in part, unspecific

- Conservative, non-invasive treatments are equally effective as more complex and invasive interventions

- From a clinical viewpoint, it may be suggested that muscle co-contracture, and not the mechanical obstacle of the displaced disc, is the key factor explaining the physiopathology of DDWoR.

1. Schiffman E, Ohrbach R, Truelove EL, et al. Diagnostic Criteria for Temporomandibular Disorders (DC/TMD) for Clinical and Research Applications: recommendations of the International RDC/TMD Consortium Network* and Orofacial Pain Special Interest Groupt. J Oral Facial Pain Headache 2014; 28: 6-27.

2. Manfredini D. Fundamentals of TMD management. In: Manfredini D (ed). Current concepts on temporomandibular disorders. Berlin, Quintessence Publishing, 2010; 305-318.

3. Manfredini D, Favero L, Gregorini G, Cocilovo F, Guarda-Nardini L. Natural course of temporomandibular disorders with low pain-related impairment: a 2-to-3-year follow-up study. J Oral Rehabil 2013; 40: 436-442.

4. Yura S. Natural course of acute closed lock of the temporomandibular joint. Br J Oral Maxillofac Surg 2012; 50: 646-649.

Evidence-Based Dentistry (2014) 15, 90-91. doi:10.1038/sj.ebd.6401049 